\title{
Generalized Coherent State Approach to Star Products and Applications to the Fuzzy Sphere
}

\author{
G. Alexanian ${ }^{a}$, A. Pinzul $^{b}$ And A. Stern ${ }^{b}$ \\ a) Department of Physics, Syracuse University, \\ Syracuse, New York 13244-1130, USA \\ b) Department of Physics, University of Alabama, \\ Tuscaloosa, Alabama 35487, USA
}

\begin{abstract}
We construct a star product associated with an arbitrary two dimensional Poisson structure using generalized coherent states on the complex plane. From our approach one easily recovers the star product for the fuzzy torus, and also one for the fuzzy sphere. For the latter we need to define the 'fuzzy' stereographic projection to the plane and the fuzzy sphere integration measure, which in the commutative limit reduce to the usual formulae for the sphere.
\end{abstract}




\section{Introduction}

The star product is an important tool for deformation quantization and noncommutative geometry. The most well studied star product is often referred to as the Moyal star product [1], [2] (For a nice review see [3].) It allows for a quantum mechanical description on phase space. In recent times it has found application in the string theory approach to noncommutative geometry. It is of particular importance for the fuzzy torus and has a simple form when acting on the periodic modes. Another star product due to Grosse and Presnajder[ [\$] is applicable to the fuzzy sphere [5], [6], [8], [7], [9], [10], and is also of current interest in string theory [1]. This star product is constructed from coherent states on $S^{2}$ and is generalizable to arbitrary coset manifolds. [12] By relying on coherent states, the property of associativity is assured. The only other requirement on the star product is that it has a proper commutative limit. For this one assumes it to be a function of a parameter, say $\hbar$, which can be Taylor expanded about $\hbar=0$. At zeroth order the star product reduces to the ordinary product, and at first order the star (or Moyal) commutator should be proportional to the Poisson bracket. The relevant issue is to find the star product associated with a given Poisson manifold. In this regard, a nontrivial constructive approach was given by Kontsevich [13] which is applicable for arbitrary Poisson structures.

The approach taken in this article is along the lines of Berezin quantization 14, 12], and relies on generalized coherent states on the complex plane developed by Man'ko, Marmo, Sudarshan and Zaccaria[15]. Associativity is once again assured, and the results can be applied to general two dimensional Poisson structures. From our approach one easily recovers the Moyal star product (or more precisely, an equivalent construction due to Voros [16], [3]), and also a star product for the fuzzy sphere. The generalized coherent states have the usual property that they form an over complete basis for an infinite dimensional Hilbert space. They are eigenstates of a 'deformed' annihilation operator. For the fuzzy sphere, we must perform a 'stereographic projection' of the usual operators generating the fuzzy sphere algebra, and a slight modification of the above procedure is necessary. This is because away from the classical limit a) the Hilbert space is finite dimensional, and a related issue is b) the deformed annihilation operator is not diagonalized by the coherent states. The deformed annihilation operator in this case is the operator analogue of the stereographic coordinate. Our result for the star product of the fuzzy sphere is expressed as an integral of hypergeometric functions. We show that it has the proper commutative limit $j \rightarrow \infty$, where $2 j+1$ is the number of dimensions ( $j=$ half-integer), and further that the fuzzy stereographic projection and fuzzy integration measure reduce to the usual formulae for the sphere in the limit. As usual, the stereographic projection has a coordinate singularity, which we choose at the north pole, in the commutative limit. However, away from $j \rightarrow \infty$, we can argue that there is no coordinate singularity, simply because the quantum mechanical probability of being at the north pole is zero. The fuzzy sphere star product of ref. [4] is expressed in terms of the three dependent coordinates of the sphere obtained from embedding it in $R^{3}$. Their product is later projected 
down to the plane via the standard stereographic projection. Ours, on the other hand, is written directly on the two dimensional domain, and may therefore be a more convenient tool for writing down noncommuting field theories. Since the fuzzy sphere is a (finite dimensional) matrix model, the corresponding field theory must be absent of any divergences, in contrast to the case of field theories on the fuzzy torus. We plan to pursue this in future works.

In the next section we give a general formula for the star product in two dimensions based on generalized coherent states. There we show how to recover the Voros product. In the general case, we find a complete set of functions which close under the action of the star product. In section 3, we apply the star product formula to the case of the fuzzy sphere and write down the fuzzy stereographic projection. The appendix shows how to express the normalization factor for the fuzzy sphere coherent states in terms of a hypergeometric function.

\section{Star Product}

\subsection{General Properties}

For our star product we introduce generalized coherent states $|\zeta\rangle$, with the label $\zeta$ corresponding to a point on the complex plane. They are assumed to form an (overcomplete) basis for Hilbert space $H$. As is usual for coherent states, they are unit vectors $\langle\zeta \mid \zeta\rangle=1$ and satisfy the completeness relation

$$
1=\int d \mu(\zeta, \bar{\zeta})|\zeta><\zeta|
$$

where $d \mu(\zeta, \bar{\zeta})$ is the appropriate measure on the complex plane, and the bar denotes complex conjugation. We also assume the existence of another basis for $H$, and in terms of this basis the states $\mid \zeta>$ are expressible in a power series in $\zeta$ times some overall normalization.

To every operator $A$ on Hilbert space $H$ one can associate a function $\mathcal{A}(\zeta, \bar{\zeta})$ on the complex plane according to

$$
\mathcal{A}(\zeta, \bar{\zeta})=<\zeta|A| \zeta>
$$

An associative product for two such functions is then defined by

$$
\begin{aligned}
\mathcal{A}(\zeta, \bar{\zeta}) \star \mathcal{B}(\zeta, \bar{\zeta}) & =\langle\zeta|A B| \zeta> \\
& =\int d \mu(\eta, \bar{\eta})<\zeta|A| \eta><\eta|B| \zeta>
\end{aligned}
$$

If $\mid \zeta>(<\zeta \mid)$ is, up to a normalization factor, analytic (anti-analytic) in $\zeta$, then the ratio $<\eta|A| \zeta>/<\eta \mid \zeta>$ is analytic in $\zeta$ and anti-analytic in $\eta$. Furthermore, it can be obtained from $\mathcal{A}(\zeta, \bar{\zeta})$ by acting with the translation operator twice

$$
e^{-\zeta \frac{\partial}{\partial \eta}} e^{\eta \frac{\partial}{\partial \zeta}} \mathcal{A}(\zeta, \bar{\zeta})=e^{-\zeta \frac{\partial}{\partial \eta}} \frac{<\zeta|A| \zeta+\eta>}{<\zeta \mid \zeta+\eta>}=\frac{<\zeta|A| \eta>}{<\zeta \mid \eta>}
$$




$$
e^{-\bar{\zeta} \frac{\partial}{\partial \bar{\eta}}} e^{\bar{\eta} \frac{\partial}{\partial \zeta}} \mathcal{A}(\zeta, \bar{\zeta})=e^{-\bar{\zeta} \frac{\partial}{\partial \bar{\eta}}} \frac{\langle\zeta+\eta|A| \zeta>}{<\zeta+\eta \mid \zeta>}=\frac{<\eta|A| \zeta>}{<\eta \mid \zeta>}
$$

Alternatively, we can write $e^{-\zeta \frac{\partial}{\partial \eta}} e^{\eta \frac{\partial}{\partial \zeta}}$ (acting on $\eta$-independent functions) as an ordered exponential

$$
: \exp (\eta-\zeta) \frac{\vec{\partial}}{\partial \zeta}:
$$

where the derivatives are ordered to the right in each term in the Taylor expansion, and they also act to the right. Similarly, we define

$$
: \exp \frac{\overleftarrow{\partial}}{\partial \zeta}(\eta-\zeta):
$$

where the derivatives are ordered to the left in each term in the Taylor expansion, and they act to the left. Substituting into (2.3), we can then write the product on functions of $\zeta$ and $\bar{\zeta}$ according to

$$
\star=\int d \mu(\eta, \bar{\eta}): \exp \frac{\overleftarrow{\partial}}{\partial \zeta}(\eta-\zeta):|<\zeta| \eta>\left.\right|^{2}: \exp (\bar{\eta}-\bar{\zeta}) \frac{\vec{\partial}}{\partial \bar{\zeta}}:
$$

The product is thus determined once we know the measure $d \mu(\zeta, \bar{\zeta})$ and the scalar product $<\zeta \mid \eta>$.

The product (2.5) has the property that in general it is not symmetric. It reduces to the ordinary product if the function on the right is analytic in $\zeta$ and the function on the left is anti-analytic in $\zeta$

$$
\mathcal{A}(\bar{\zeta}) \star \mathcal{B}(\zeta)=\mathcal{A}(\bar{\zeta}) \mathcal{B}(\zeta)
$$

If we have that the states $\mid \zeta>$ are eigenvectors of some operator $\tilde{\mathbf{a}}$ with eigenvalues $\zeta$

$$
\tilde{\mathbf{a}}|\zeta>=\zeta| \zeta>
$$

then the product $(2.5)$ between two analytic functions also reduces to the ordinary product

$$
\begin{aligned}
\mathcal{A}(\zeta) \star \mathcal{B}(\zeta) & =\langle\zeta|\mathcal{A}(\tilde{\mathbf{a}})| \zeta>\star\langle\zeta|\mathcal{B}(\tilde{\mathbf{a}})| \zeta> \\
& =\langle\zeta|\mathcal{A}(\tilde{\mathbf{a}}) \mathcal{B}(\tilde{\mathbf{a}})| \zeta> \\
& =\mathcal{A}(\zeta) \mathcal{B}(\zeta)
\end{aligned}
$$

Similarly, then the product (2.5) between two anti-analytic functions reduces to the ordinary product

$$
\mathcal{A}(\bar{\zeta}) \star \mathcal{B}(\bar{\zeta})=\mathcal{A}(\bar{\zeta}) \mathcal{B}(\bar{\zeta})
$$

For this we only need $<\zeta\left|\tilde{\mathbf{a}}^{\dagger}=\bar{\zeta}<\zeta\right|$. To get nontrivial results we can take the function on the right to be anti-analytic in $\zeta$ and the function on the left to be analytic in $\zeta$

$$
\begin{aligned}
\mathcal{A}(\zeta) \star \mathcal{B}(\bar{\zeta}) & =\left\langle\zeta\left|\mathcal{A}(\tilde{\mathbf{a}}) \mathcal{B}\left(\tilde{\mathbf{a}}^{\dagger}\right)\right| \zeta>\right. \\
& =\mathcal{A}(\zeta) \mathcal{B}(\bar{\zeta})+<\zeta\left|\left[\mathcal{A}(\tilde{\mathbf{a}}), \mathcal{B}\left(\tilde{\mathbf{a}}^{\dagger}\right)\right]\right| \zeta>
\end{aligned}
$$


which can be evaluated once we know the commutation relations for $\tilde{\mathbf{a}}$ and $\tilde{\mathbf{a}}^{\dagger}$. Say the commutation relations are of the form

$$
\left[\tilde{\mathbf{a}}, \tilde{\mathbf{a}}^{\dagger}\right]=F\left(\tilde{\mathbf{a}} \tilde{\mathbf{a}}^{\dagger}\right)
$$

for some function $F$, and $F$ can be Taylor expanded in some (commuting) parameter $\hbar$, where the lowest order term is linear in $\hbar$. For the "classical limit" defined as $\hbar \rightarrow 0$, one demands that

$$
\begin{aligned}
\mathcal{A}(\zeta, \bar{\zeta}) \star \mathcal{B}(\zeta, \bar{\zeta}) & \rightarrow \mathcal{A}(\zeta, \bar{\zeta}) \mathcal{B}(\zeta, \bar{\zeta}) \\
\mathcal{A}(\zeta, \bar{\zeta}) \star \mathcal{B}(\zeta, \bar{\zeta})-\mathcal{B}(\zeta, \bar{\zeta}) \star \mathcal{A}(\zeta, \bar{\zeta}) & \rightarrow O(\hbar)
\end{aligned}
$$

and the coefficient on the right hand side of (2.13) is identified with the Poisson bracket. [For the star product of an analytic function with an anti-analytic function, these two conditions reduce to one: $\mathcal{A}(\zeta) \star \mathcal{B}(\bar{\zeta}) \rightarrow \mathcal{A}(\zeta) \mathcal{B}(\bar{\zeta})+O(\hbar)$ thanks to (2.6) .] We thereby obtain all the properties of the star product.

\subsection{Standard Coherent States}

From the standard coherent states it is easy to recover the Moyal[1], [2] (or actually the Voros [16]) star product. f Here we identify $\tilde{\mathbf{a}}$ and $\tilde{\mathbf{a}}^{\dagger}$ with the standard lowering and raising operators for the harmonic oscillator $\mathbf{a}$ and $\mathbf{a}^{\dagger}$, satisfying

$$
\left[\mathbf{a}, \mathbf{a}^{\dagger}\right]=1
$$

(For the moment we supress $\hbar$.) Then products such as (2.10) are easy to compute. It is also easy to perform the integral in (2.5) in this case. The scalar product squared is $|\langle\zeta \mid \eta\rangle|^{2}=$ $e^{-|\zeta-\eta|^{2}}$ and the measure is

$$
d \mu(\eta, \bar{\eta})=\frac{1}{\pi} d \eta_{R} d \eta_{I}
$$

$\eta_{R}$ and $\eta_{I}$ being the real and imaginary parts of $\eta$. The integrand in (2.21) is then a Gaussian:

$$
\begin{aligned}
\star & =\frac{1}{\pi} \int d \eta_{R} d \eta_{I} \quad: \exp \frac{\overleftarrow{\partial}}{\partial \zeta}(\eta-\zeta): \exp \left(-|\zeta-\eta|^{2}\right) \quad: \exp (\bar{\eta}-\bar{\zeta}) \frac{\vec{\partial}}{\partial \bar{\zeta}}: \\
& =\frac{1}{\pi} \int d \eta_{R} d \eta_{I} \exp \frac{\overleftarrow{\partial}}{\partial \zeta} \eta \exp \left(-|\eta|^{2}\right) \exp \bar{\eta} \frac{\vec{\partial}}{\partial \bar{\zeta}} \\
& =\exp \frac{\overleftarrow{\partial}}{\partial \zeta} \frac{\vec{\partial}}{\partial \bar{\zeta}}
\end{aligned}
$$

Note that the ordering of the exponential function can be dropped after the change of integration variables. For this to be a star product it must contain a parameter (say $\hbar$ ) which admits

*After posting an earlier version of this article on the hep-th archive, we were informed of a similar discussion in 117 . 
a commutative limit $(\hbar \rightarrow 0)$. This is easily done by a rescaling of the coordinates $\zeta \rightarrow \frac{1}{\sqrt{\hbar}} \zeta$ and then

$$
\star \rightarrow \exp \hbar \frac{\overleftarrow{\partial}}{\partial \zeta} \frac{\partial}{\partial \bar{\zeta}}
$$

This is the star product of Voros [16], which is equivalent to the standard star product on the plane [1], namely

$$
\tilde{\star}=\exp \frac{\hbar}{2}\left(\frac{\overleftarrow{\partial}}{\partial \zeta} \frac{\vec{\partial}}{\partial \bar{\zeta}}-\frac{\overleftarrow{\partial}}{\partial \bar{\zeta}} \frac{\vec{\partial}}{\partial \zeta}\right)
$$

The equivalence relation is $T(\mathcal{A}) \star T(\mathcal{B})=T(\mathcal{A} \tilde{\star} \mathcal{B})$, where $T$ is a nonsingular operator. Using the identity

$$
\exp \frac{\hbar}{2} \frac{\partial}{\partial \zeta} \frac{\partial}{\partial \bar{\zeta}}(\mathcal{A B})=\mathcal{A} \exp \frac{\hbar}{2}\left(\frac{\overleftarrow{\partial}}{\partial \zeta} \frac{\overleftarrow{\partial}}{\partial \bar{\zeta}}+\frac{\vec{\partial}}{\partial \bar{\zeta}} \frac{\vec{\partial}}{\partial \zeta}+\frac{\overleftarrow{\partial}}{\partial \zeta} \frac{\vec{\partial}}{\partial \bar{\zeta}}+\overleftarrow{\frac{\partial}{\partial \bar{\zeta}}} \frac{\vec{\partial}}{\partial \zeta}\right) \mathcal{B}
$$

it can be easily checked that the relevant operator is[3]

$$
T=\exp \frac{\hbar}{2} \frac{\partial}{\partial \zeta} \frac{\partial}{\partial \bar{\zeta}}
$$

(An alternative connection between coherent states and the Moyal star product is deduced in [18], [19].)

\subsection{Deformed Coherent States}

A more general class of coherent states on the complex plane was given in [15]. These coherent states provide a more convenient basis than the standard coherent states when studying functions of operators $\tilde{\mathbf{a}}$ and $\tilde{\mathbf{a}}^{\dagger}$. Now we assume such operators satisfy the general commutation relations (2.11), while the coherent states satisfy (2.7). The expectation values of $\tilde{\mathbf{a}}$ and $\tilde{\mathbf{a}}^{\dagger}$ for the coherent state $\mid \zeta>$ are $\zeta$ and $\bar{\zeta}$, respectively, and the star product (2.5) can be directly applied to functions of these variables.

The procedure of [15] requires the existence of a map from the usual harmonic oscillator algebra generated by annihilation and creation operators $\mathbf{a}$ and $\mathbf{a}^{\dagger}$, satisfying (2.14), to the algebra generated by $\tilde{\mathbf{a}}$ and $\tilde{\mathbf{a}}^{\dagger}$. The map is expressed in the form

$$
\tilde{\mathbf{a}}=f(\mathbf{n}+1) \mathbf{a},
$$

$\mathbf{n}$ being the number operator $\mathbf{n}=\mathbf{a}^{\dagger} \mathbf{a}$, and the function $f$ being determined from $F$. In this section we regard $f(\mathbf{n})$ as a nonsingular function, while we adapt the formalism to a singular function in the section 3. Following [15] we restrict to real functions, as only the real part of $f$ determines $F$. We can introduce the Hilbert space $\mathrm{H}$ spanned by orthonormal states $|n\rangle$, $n=0,1,2, \ldots$, with $\mathbf{a} \mid 0>=0$ and $\mathbf{n}|n>=n| n>$. Following [15] one can construct the analogue of the standard coherent states according to:

$$
\left|\zeta>=N\left(|\zeta|^{2}\right)^{-\frac{1}{2}} \exp \left\{\zeta f(\mathbf{n})^{-1} \mathbf{a}^{\dagger}\right\} f(\mathbf{n})^{-1}\right| 0>
$$




$$
=N\left(|\zeta|^{2}\right)^{-\frac{1}{2}} \sum_{n=0}^{\infty} \frac{\zeta^{n}}{\sqrt{n !}[f(n)] !} \mid n>
$$

where $[f(n)] !=f(n) f(n-1) \ldots f(0)$. These states are diagonal in $\tilde{\mathbf{a}}$, rather than $\mathbf{a}$, with associated eigenvalues $\zeta$ as in (2.7). Requiring them to be of unit norm fixes $N\left(|\zeta|^{2}\right.$ ),

$$
N(x)=\sum_{n=0}^{\infty} \frac{x^{n}}{n !([f(n)] !)^{2}},
$$

which reduces to the exponential function for standard coherent states. As with the standard coherent states, the states (2.18) are not orthonormal. Now

$$
<\eta \mid \zeta>=N\left(|\eta|^{2}\right)^{-\frac{1}{2}} N\left(|\zeta|^{2}\right)^{-\frac{1}{2}} N(\bar{\eta} \zeta)
$$

Substituting into (2.5) then gives

$$
\star=\int d \mu(\eta, \bar{\eta}): \exp \frac{\overleftarrow{\partial}}{\partial \zeta}(\eta-\zeta): \frac{N(\bar{\eta} \zeta) N(\bar{\zeta} \eta)}{N\left(|\eta|^{2}\right) N\left(|\zeta|^{2}\right)}: \exp (\bar{\eta}-\bar{\zeta}) \frac{\vec{\partial}}{\partial \bar{\zeta}}:
$$

From (2.20) and the completeness relation, the integration measure should satisfy

$$
N(\bar{\zeta} \lambda)=\int d \mu(\eta, \bar{\eta}) \frac{N(\bar{\zeta} \eta) N(\bar{\eta} \lambda)}{N\left(|\eta|^{2}\right)}
$$

for arbitrary complex coordinates $\zeta$ and $\lambda$. If we assume that it is of the form $d \mu(\zeta, \bar{\zeta})=$ $i h\left(|\zeta|^{2}\right) d \zeta \wedge d \bar{\zeta}$, then the conditions on $h\left(|\zeta|^{2}\right)$ are

$$
\int_{0}^{\infty} \frac{d \rho \rho^{2 n+1} h\left(\rho^{2}\right)}{N\left(\rho^{2}\right)}=\frac{n !}{4 \pi}([f(n)] !)^{2}, \quad \text { for all integer } n \geq 0 .
$$

Upon defining $g(x)=h(x) / N(x)$, we can rewrite (2.23) as

$$
\int_{0}^{\infty} d x x^{s-1} g(x)=\frac{\Gamma(s)}{2 \pi}([f(s-1)] !)^{2}, \quad \text { for all integer } s \geq 1 .
$$

By definition the right hand side is the Mellin transformation of $g(x)$. Then $g(x)$ can be written as inverse Mellin integral transform

$$
g(x)=\frac{1}{2 \pi i} \int_{c-i \infty}^{c+i \infty} \frac{\Gamma(s)}{2 \pi}([f(s-1)] !)^{2} x^{-s} d s
$$

where here $s$ is treated as a complex integration variable (with some possible restrictions) and it is assumed that the function $[f(s-1)]$ ! can be extended appropriately over the entire integration region. For the case of the standard coherent states where $f(s)=1$, we then get that $g(x)=e^{-x} / 2 \pi$. Taking into account that for standard case $N(x)=e^{x}$ we recover the integration measure (2.15). 
It is possible to compute the star product for a class of function without using a specific expression for measure. For example, $1 \star 1=1$, which follows from (2.22). Furthermore, using (2.23), for the functions

$$
\mathcal{Z}_{n m}=\frac{\bar{\zeta}^{n} \zeta^{m}}{N\left(|\zeta|^{2}\right)}, \quad n, m=0,1,2,3, \ldots
$$

with the property $\overline{\mathcal{Z}}_{n m}=\mathcal{Z}_{m n}$, the unit function acts as the identity with respect to the the star product: $1 \star \mathcal{Z}_{n m}=\mathcal{Z}_{n m} \star 1=\mathcal{Z}_{n m}$, and these functions form a closed algebra:

$$
\mathcal{Z}_{n m} \star \mathcal{Z}_{r s}=m !([f(m)] !)^{2} \delta_{m, r} \mathcal{Z}_{n s}
$$

This algebra has the projectors

$$
P_{m}=\frac{\mathcal{Z}_{m m}}{m !([f(m)] !)^{2}}
$$

The square (using $\star$ ) of all other functions vanishes. We can write $\zeta$ and $\bar{\zeta}$ in terms of of functions $\mathcal{Z}_{n m}$

$$
\zeta=\sum_{n=0}^{\infty} \frac{\mathcal{Z}_{n n+1}}{n !([f(n)] !)^{2}}, \quad \bar{\zeta}=\sum_{n=0}^{\infty} \frac{\mathcal{Z}_{n+1 n}}{n !([f(n)] !)^{2}},
$$

and thereby compute their star products:

$$
\begin{aligned}
\bar{\zeta} \star \zeta & =|\zeta|^{2} \\
\zeta \star \zeta & =\zeta^{2} \\
\bar{\zeta} \star \bar{\zeta} & =\bar{\zeta}^{2} \\
\zeta \star \bar{\zeta} & =|\zeta|^{2}+<\zeta\left|F\left(\tilde{\mathbf{a}} \tilde{a}^{\dagger}\right)\right| \zeta> \\
<\zeta\left|F\left(\tilde{\mathbf{a}} \tilde{\mathbf{a}}^{\dagger}\right)\right| \zeta>= & \sum_{n=0}^{\infty}\left((n+1) f(n+1)^{2}-n f(n)^{2}\right) P_{n}
\end{aligned}
$$

which is consistent with $(2.62 .10)$.

\section{4 q-oscillators}

Coherent states for q-oscillators were studied by many authors [22, 223, 224]. The q-oscillators algebra is generated by $\tilde{\mathbf{a}}, \tilde{\mathbf{a}}^{\dagger}$ and $\mathbf{n}$ satisfying the algebra 25, 26]

$$
\begin{gathered}
\tilde{\mathbf{a}}^{\dagger}-q^{-1} \tilde{\mathbf{a}}^{\dagger} \tilde{\mathbf{a}}=q^{\mathbf{n}} \\
{[\mathbf{n}, \tilde{\mathbf{a}}]=-\tilde{\mathbf{a}}, \quad\left[\mathbf{n}, \tilde{\mathbf{a}}^{\dagger}\right]=\tilde{\mathbf{a}}^{\dagger},}
\end{gathered}
$$

where we consider $q$ to be a real parameter, and when $q \rightarrow 1$ we recover the ordianry oscillator algebra. As the notation implies $\mathbf{n}$ can be identified with the 'undeformed' number operator $\mathbf{n}=\mathbf{a}^{\dagger} \mathbf{a}$, while $f$ in $(2.17)$ is given by

$$
f(n)^{2}=\frac{1}{n} \frac{q^{n}-q^{-n}}{q-q^{-1}} \equiv \frac{[n]}{n},
$$


and $N(x)$ corresponds to a q-exponential function,

$$
N(x)=\sum_{n=0}^{\infty} \frac{x^{n}}{[n] !} \equiv e_{q}(x),
$$

$[n] !=[n][n-1] \ldots[0]$. According to [22], for the measure one can use

$$
h\left(\rho^{2}\right) d \rho^{2}=\frac{1}{2 \pi} e_{q}\left(\rho^{2}\right) e_{q}\left(-\rho^{2}\right) d_{q} \rho^{2},
$$

where the 'q-integration' over $\rho^{2}$ is defined by

$$
\int_{0}^{\varsigma} g(x) d_{q} x=\varsigma\left(q^{-1 / 2}-q^{1 / 2}\right) \sum_{n=0}^{\infty} q^{n+1 / 2} g\left(\varsigma q^{n+1 / 2}\right),
$$

and $-\varsigma$ is the largest zero of $e_{q}(x)$.

\section{$3 \quad$ Fuzzy sphere}

With a small modification of the above procedure we can write down the star product for the fuzzy sphere. The modification is necessary because we will no longer have (2.7), except in the commutative limit. We associate deformed annihilation and creation operators, $\tilde{\mathbf{a}}$ and $\tilde{\mathbf{a}}^{\dagger}$, with the operator analogue of the stereographic coordinates of a sphere. Its algebra now leads to a highest weight state $\mid 2 j>$ and therefore finite $(2 j+1)$ dimensional representations, which is another departure from the treatment of the previous section. It requires that we terminate the series in (2.18) and what follows, and quantities computed previously now depend on $j$. The infinite series is recovered when $j \rightarrow \infty$, which is the commutative limit of the fuzzy sphere.

For finite $j$, we are able to obtain exact expressions for the normalization factor $N_{j}\left(|\zeta|^{2}\right)$ and integration measure $d \mu_{j}(\zeta, \bar{\zeta})$ in terms of hypergeometric functions.

\subsection{Fuzzy Stereographic Projection}

We first recall that the stereographic projection of a sphere of radius $1, x_{i} x_{i}=1, i=1,2,3$, to the complex plane which maps the north pole to infinity is given by

$$
z=\frac{x_{1}-i x_{2}}{1-x_{3}}, \quad \bar{z}=\frac{x_{1}+i x_{2}}{1-x_{3}}
$$

To obtain the algebra of the fuzzy sphere one promotes the coordinates $x_{i}$ to operators $\mathbf{x}_{i}$ 's , satisfying commutation relations:

$$
\left[\mathbf{x}_{i}, \mathbf{x}_{j}\right]=i \alpha \epsilon_{i j k} \mathbf{x}_{k}
$$

as well as $\mathbf{x}_{i} \mathbf{x}_{i}=1$, where $\alpha$ is a parameter which vanishes in the commutative limit and 1 now denotes the unit operator. For $\alpha=\frac{1}{\sqrt{j(j+1)}}, j=\frac{1}{2}, 1, \frac{3}{2}, \ldots, \mathbf{x}_{i}$ has finite dimensional 
representations, which are simply given by $\mathbf{x}_{i}=\alpha \mathbf{J}_{i}, \mathbf{J}_{i}$ being the angular momentum matrices. To define an operator analogue of the stereographic projection of operators $\mathbf{x}_{i}$, we need to choose an ordering in the definition of operators $\mathbf{z}$ and $\mathbf{z}^{\dagger}$. We define the following deformation map of the algebra:

$$
\mathbf{z}=\left(\mathbf{x}_{1}-i \mathbf{x}_{2}\right)\left(1-\mathbf{x}_{3}\right)^{-1}, \quad \mathbf{z}^{\dagger}=\left(1-\mathbf{x}_{3}\right)^{-1}\left(\mathbf{x}_{1}+i \mathbf{x}_{2}\right)
$$

From the commutation relations (3.40) one gets

$$
\left[\mathbf{z}, \chi^{-1}\right]=-\frac{\alpha}{2} \mathbf{z}, \quad\left[\mathbf{z}^{\dagger}, \chi^{-1}\right]=\frac{\alpha}{2} \mathbf{z}^{\dagger}
$$

where $\chi^{-1}=\frac{1}{2}\left(1-\mathbf{x}_{3}\right)$. It then follows that $\chi^{-1}$ commutes with $|\mathbf{z}|^{2}=\mathbf{z z}^{\dagger}$ and

$$
\left[\mathbf{z}, \mathbf{z}^{\dagger}\right]=\alpha \chi\left(1+|\mathbf{z}|^{2}-\frac{1}{2} \chi\left(1+\frac{\alpha}{2}|\mathbf{z}|^{2}\right)\right)
$$

This is the analogue of eq. (2.11), the right hand side corresponding to the function $F$. For all finite dimensional matrix representations of the fuzzy sphere, $\chi^{-1}$ is represented by a nonsingular matrix, and the above equation makes sense. More generally, $\chi^{-1}$ is a nonsingular operator. Since it is a hermitian operator, and it should be possible to write it in terms of $|\mathbf{z}|^{2}$. To get its form start with the identity

$$
\mathbf{z} \chi^{-2} \mathbf{z}^{\dagger}+\chi^{-1} \mathbf{z}^{\dagger} \mathbf{z} \chi^{-1}+2 \chi^{-1}\left(\chi^{-1}-1\right)=0
$$

which follows from $\mathbf{x}_{i} \mathbf{x}_{i}=1$, and apply the above commutation relations to get

$$
\frac{\alpha}{4} \xi \chi^{2}-\chi\left(\xi+\frac{\alpha}{2}\right)+1+|\mathbf{z}|^{2}=0, \quad \xi=1+\alpha|\mathbf{z}|^{2}
$$

having the solution

$$
\frac{\alpha}{2} \chi=1+\frac{\alpha}{2 \xi}-\sqrt{\frac{1}{\xi}+\left(\frac{\alpha}{2 \xi}\right)^{2}}
$$

The sign choice is so that $\chi$ reduces to $1+|\mathbf{z}|^{2}$ in the limit $j \rightarrow \infty$, and thus the right hand side of (3.42) goes to

$$
\frac{1}{2 j}\left(1+|\mathbf{z}|^{2}\right)^{2}
$$

Since for finite $j$, the eigenvalues of $\left(1+\frac{\alpha}{2 \xi}\right)^{2}$ are greater than those of $\frac{1}{\xi}+\left(\frac{\alpha}{2 \xi}\right)^{2}$, it follows that $\chi$ is an invertable operator. There is thus a $1-1$ correspondence between representations of the algebra generated by $\mathbf{z}$ and $\mathbf{z}^{\dagger}$ and the algebra of the fuzzy sphere.

One of the attractive features of the fuzzy sphere as a noncommutative space is that it is covariant with respect to the same symmetry as the standard sphere, namely $S O(3)$. (This is in contrast to the case of the quantum sphere. 20]) Upon stereographically projecting the symmetry transformations of the standard sphere to the complex plane one gets elements of the Mobius group. These are nonlinear transformations, so in the case of the fuzzy sphere, we 
have to be concerned with operator orderings. For infinitesimal rotations of the fuzzy sphere, $\delta \mathbf{x}_{i}=\epsilon_{i j k} \epsilon_{j} \mathbf{x}_{k}, \epsilon_{j}$ being an infinitesimal c-number, $\mathbf{z}$ and $\mathbf{z}^{\dagger}$ undergo the variations

$$
\begin{aligned}
\delta \mathbf{z} & =-i \epsilon_{3} \mathbf{z}-\frac{\epsilon_{-}}{2} \mathbf{z}^{2}+\frac{\epsilon_{+}}{2}\left(\chi-2-\frac{1}{4} \mathbf{z} \chi \mathbf{z}^{\dagger} \chi\right), \\
\delta \mathbf{z}^{\dagger} & =i \epsilon_{3} \mathbf{z}^{\dagger}-\frac{\epsilon_{+}}{2} \mathbf{z}^{\dagger}+\frac{\epsilon_{-}}{2}\left(\chi-2-\frac{1}{4} \chi \mathbf{z} \chi \mathbf{z}^{\dagger}\right),
\end{aligned}
$$

where $\epsilon_{ \pm}=\epsilon_{2} \pm i \epsilon_{1}$.

For the two, three and four dimensional representations of $\mathbf{z}$ and $|\mathbf{z}|^{2}$, we get, respectively,

$$
\begin{aligned}
& \mathbf{z}=\left(\begin{array}{cc}
0 & 0 \\
1+\sqrt{3} & 0
\end{array}\right) \\
& |\mathbf{z}|^{2}=\operatorname{diag}(0,2(2+\sqrt{3})) \\
& \mathbf{z}=\left(\begin{array}{ccc}
0 & 0 & 0 \\
2+\sqrt{2} & 0 & 0 \\
0 & 1 & 0
\end{array}\right) \\
& |\mathbf{z}|^{2}=\operatorname{diag}(0,2(3+2 \sqrt{2}), 1) \\
& \mathbf{z}=\left(\begin{array}{cccc}
0 & 0 & 0 & 0 \\
\sqrt{3}+\sqrt{5} & 0 & 0 & 0 \\
0 & \frac{2}{7}(1+\sqrt{15}) & 0 & 0 \\
0 & 0 & \frac{1}{7}(3 \sqrt{5}-\sqrt{3}) & 0
\end{array}\right) \\
& |\mathbf{z}|^{2}=\operatorname{diag}\left(0,2(4+\sqrt{15}), \frac{8}{49}(8+\sqrt{15}), \frac{6}{49}(8-\sqrt{15})\right)
\end{aligned}
$$

More generally, we denote the states of an irreducible representation $\Gamma^{j}$ as usual by $\mid j, m>$, $j=\frac{1}{2}, 1, \frac{3}{2}, \ldots, m=-j,-j+1, \ldots, j$. The states span vector space $H^{j}$. Then $|\mathbf{z}|^{2} \mid j, m>=$ $\lambda_{j, m} \mid j, m>$, with

$$
\lambda_{j, m}=\frac{j(j+1)-m(m+1)}{(\sqrt{j(j+1)}-m-1)^{2}} .
$$

As $j \rightarrow \infty, \lambda_{j, m}$ ranges between 0 and $8 j+4$.

\subsection{Coherent States and Star Product}

We next define the map from the harmonic oscillator algebra. This is clearly a singular map since $H^{j}$ is finite dimensional and the Hilbert space $H$ for the latter is not. For irreducible representation $\Gamma^{j}$, we can restrict the map to act on the finite dimensional subspace of $\mathrm{H}$ spanned by the first $2 j+1$ states $\mid n>, n=0,1,2, \ldots, 2 j$. More precisely, we identify $\mid j, m>$ 
in $H^{j}$ with $\mid j+m>$ of $\mathrm{H}$, and the map is applied to this subspace. Because the map depends on $j$ we include a $j$ subscript

$$
\mathbf{z}=f_{j}(\mathbf{n}+1) \mathbf{a}
$$

From (3.42) and (2.14) the eigenvalues of $f_{j}(\mathbf{n})^{2}$ in $H^{j}$ are $\lambda_{j, n-j-1} / n$. Therefore,

$$
f_{j}(\mathbf{n})=\frac{\sqrt{2 j-\mathbf{n}+1}}{\sqrt{j(j+1)}+j-\mathbf{n}}
$$

It is zero when acting on $\mid 2 j+1>$ and hence $\mathbf{z}^{\dagger} \mid 2 j>=0$. It is ill-defined for harmonic oscillator states with $n>2 j+1$. The map (3.49) is similar to that of Holstein and Primakoff[21], who instead go from the angular momentum algebra to the oscillator algebra.

We now construct coherent states, as before, with a linear combination of $\mathbf{n}$ eigenstates. Only here we need to truncate the series at $n=2 j$ :

$$
\left|\zeta, j>=N_{j}\left(|\zeta|^{2}\right)^{-\frac{1}{2}} \sum_{n=0}^{2 j} \frac{\zeta^{n}}{\sqrt{n !}\left[f_{j}(n)\right] !}\right| n>.
$$

The normalization condition is now

$$
N_{j}(x)=\sum_{n=0}^{2 j} \frac{x^{n}}{n !\left(\left[f_{j}(n)\right] !\right)^{2}},
$$

which can be expressed in terms of a hypergeometric function [see appendix]

$$
N_{j}(x)=\frac{\Gamma(\gamma+2 j+1)^{2}}{(2 j+1) !(2 j) ! \Gamma(\gamma)^{2}}{ }_{3} F_{2}\left(1,1,-2 j ; \gamma, \gamma ;-x^{-1}\right) x^{2 j}
$$

where $\gamma=\sqrt{j(j+1)}-j$. To calculate the integration measure according to the general formula (2.25) we use the Mellin-Barnes type integral representation of hypergeometric function ${ }_{2} F_{1}$

$$
\frac{\Gamma(a) \Gamma(b)}{\Gamma(c)}{ }_{2} F_{1}(a, b ; c ;-z)=\frac{1}{2 \pi i} \int_{-i \infty}^{i \infty} \frac{\Gamma(a-s) \Gamma(b-s) \Gamma(s)}{\Gamma(c-s)} z^{-s} d s, \quad|\arg (z)|<\pi,
$$

where the path of integration is such that all poles due to $\Gamma(a-s)$ and $\Gamma(b-s)$ lie to the right of the path. Then using (2.25) we have

$$
h_{j}(x)=\frac{N_{j}(x)}{2 \pi}{ }_{2} F_{1}(\gamma+2 j+1, \gamma+2 j+1 ; 2 j+2 ;-x) .
$$

This result is valid for $0<s<\gamma+2 j+1$ in (2.24), or $-1<n<\gamma+2 j$ in (2.23), which contains the compete set of eigenvalues of the number operator $\mathbf{n}$. The integration measure $d \mu_{j}(\zeta, \bar{\zeta})$ is a product of hypergeometric functions, and thus the star product 2.21) for the fuzzy sphere can now be given as an integral of hypergeometric functions.

For finite $j$ the coherent states (3.51) are not diagonal in $\mathbf{z}$. (z has only zero eigenvalues for all finite $j$.) Instead,

$$
\mathbf{z}|\zeta, j>=\zeta| \zeta, j>-\frac{N_{j}\left(|\zeta|^{2}\right)^{-\frac{1}{2}} \zeta^{2 j+1}}{\sqrt{(2 j) !}\left[f_{j}(2 j)\right] !} \mid 2 j>.
$$


So as indicated earlier, we don't have the analogue of (2.7). On the other hand, $\mid \zeta, j>$ tend to $\mathbf{z}$ eigenstates in the commutative limit $j \rightarrow \infty$. For this we need the asymptotic behavior of $N_{j}(x)$. The result for $x \equiv|\zeta|^{2} \ll j$ is [see appendix]

$$
N_{j}(x) \sim(1+x)^{2 j}\left(\frac{2 j}{1+x}\right)^{2(1-\gamma)} \exp \left(\frac{1+x}{8 j}\right)
$$

From this and $\sqrt{(2 j) !}\left[f_{j}(2 j)\right] ! \sim \sqrt{2 \pi j}$, the last term in 3.55$)$ vanishes and so $\mathbf{z}$ has eigenvalue $\zeta$ in this limit. We thus expect that $\zeta$ and $\bar{\zeta}$ tend to the usual stereographic coordinates $z$ and $\bar{z}$ of the commutative sphere in this limit. This will be demonstrated explicitly later. Using the asymptotic expansion of ${ }_{2} F_{1}$ for large parameters [27]

$$
{ }_{2} F_{1}\left(a_{1}+2 j, a_{2}+2 j ; b+2 j ;-x\right) \sim(1+x)^{b-a_{1}-a_{2}-2 j}\left(1+O\left(j^{-1}\right)\right), \quad x \ll j,
$$

and the above expansion (3.56) for $N_{j}(x)$, one can find behavior of the measure for large $j$ in terms of these variables

$$
d \mu_{j}(\zeta, \bar{\zeta}) \sim \frac{j}{\pi} \frac{i d \zeta \wedge d \bar{\zeta}}{\left(1+|\zeta|^{2}\right)^{2}}, \quad x \ll j
$$

So we recover the usual measure for $S^{2}$. (We can rescale the coordinates to absorb the $j$ factor.)

From (3.56) we can make the observation that if $x$ is 'small', more precisely of order $1 / j$, then $N_{j}(x)$ goes like $2 j e^{y}$, where $y$ is a rescaled variable, $x=y /(2 j-1)$. Small $x$ corresponds to a large radius for the sphere (instead of 1 ). It thus makes sense that in this limit we recover the normalization factor associated with standard coherent states.

We now compute some star products. For the variables $\zeta$ and $\bar{\zeta}$ :

$$
\begin{aligned}
\bar{\zeta} \star \zeta & =|\zeta|^{2} \\
\zeta \star \zeta & =\zeta^{2}+\frac{\mathcal{Z}_{2 j 2 j+2}}{(2 j) !\left[f_{j}(2 j) !\right]^{2}}, \\
\bar{\zeta} \star \bar{\zeta} & =\bar{\zeta}^{2}+\frac{\mathcal{Z}_{2 j+2} 2 j}{(2 j) !\left[f_{j}(2 j) !\right]^{2}}, \\
\zeta \star \bar{\zeta} & =|\zeta|^{2}+<\zeta\left|\left[\mathbf{z}, \mathbf{z}^{\dagger}\right]\right| \zeta>-\frac{\mathcal{Z}_{2 j+12 j+1}}{(2 j) !\left[f_{j}(2 j) !\right]^{2}}
\end{aligned}
$$

The first equation agrees with (2.30), while the others contain correction terms to 2.31 2.33). These correction terms are due to the fact that the coherent states are not eigenvectors of $\mathbf{z}$, except in the commutative limit. Actually, rather than $\zeta$ and $\bar{\zeta}$, a more usefull set of variables are the 'fuzzy' stereographic coordinates $z_{F}$ and $\bar{z}_{F}$ defined by:

$$
z_{F}=\left\langle\zeta|\mathbf{z}| \zeta>, \quad \bar{z}_{F}=\left\langle\zeta\left|\mathbf{z}^{\dagger}\right| \zeta>.\right.\right.
$$

Using (3.55), they are related to $\zeta$ and $\bar{\zeta}$ by

$$
z_{F}=\zeta-\frac{\mathcal{Z}_{2 j 2 j+1}}{(2 j) !\left(\left[f_{j}(2 j)\right] !\right)^{2}}=\sum_{n=0}^{2 j-1} \frac{\mathcal{Z}_{n n+1}}{n !\left(\left[f_{j}(n)\right] !\right)^{2}},
$$




$$
\bar{z}_{F}=\bar{\zeta}-\frac{\mathcal{Z}_{2 j+12 j}}{(2 j) !\left(\left[f_{j}(2 j)\right] !\right)^{2}}=\sum_{n=0}^{2 j-1} \frac{\mathcal{Z}_{n+1 n}}{n !\left(\left[f_{j}(n)\right] !\right)^{2}},
$$

$\mathcal{Z}_{m n}$ being defined in (2.26). The transformation from $\zeta$ and $\bar{\zeta}$ to $z_{F}$ and $\bar{z}_{F}$ is nonsingular. This is because

$$
0<\frac{|\zeta|^{2 j}}{N\left(|\zeta|^{2}\right)(2 j) !\left(\left[f_{j}(2 j)\right] !\right)^{2}} \leq 1 .
$$

Also, $z_{F}$ and $\bar{z}_{F}$ tend to $\zeta$ and $\bar{\zeta}$ in the commutative limit, and just as with the latter variables, the star products of $z_{F}$ and $\bar{z}_{F}$ reduce to the ordinary products in the limit. For the star commutator of $z_{F}$ with $\bar{z}_{F}$ we can use the definition

$$
z_{F} \star \bar{z}_{F}-\bar{z}_{F} \star z_{F}=<\zeta\left|\left[\mathbf{z}, \mathbf{z}^{\dagger}\right]\right| \zeta>
$$

For $j \rightarrow \infty$ we can replace the commutator by (3.43) and the right hand side reduces to $\frac{1}{2 j}\left(1+\left|z_{F}\right|^{2}\right)^{2}$, corresponding to the Poisson bracket of $z_{F}$ and $\bar{z}_{F}$.

For finite $j, \mathcal{Z}_{n m}, n, m=0,1,2, \ldots 2 j$ generate a $(2 j+1)^{2}$ dimensional algebra given by (2.27). This algebra is isomorphic to the algebra of $(2 j+1) \times(2 j+1)$ matrices associated with the $j^{\text {th }}$ representation of the fuzzy sphere. The latter are generated by $(2 j+1) \times(2 j+1)$ matrix representations for $\mathbf{z}$ and $\mathbf{z}^{\dagger}$. For the case where $j=\frac{1}{2}$, the normalization factor is $N_{\frac{1}{2}}(x)=\left(\lambda_{\frac{1}{2},-\frac{1}{2}}+x\right) / 8, \lambda_{j, m}$ given in (3.48), while the coherent states are

$$
\mid \zeta, \frac{1}{2}>=\frac{\lambda_{\frac{1}{2},-\frac{1}{2}}^{\frac{1}{2}}|0>+\zeta| 1>}{\sqrt{\lambda_{\frac{1}{2},-\frac{1}{2}}+|\zeta|^{2}}}
$$

For the functions $\mathcal{Z}_{n m}, n, m=0,1$ in 2.26 one gets the star products

$$
\mathcal{Z}_{n 0} \star \mathcal{Z}_{0 s}=\frac{8}{\lambda_{\frac{1}{2},-\frac{1}{2}}} \mathcal{Z}_{n s}, \quad \mathcal{Z}_{n 1} \star \mathcal{Z}_{1 s}=8 \mathcal{Z}_{n s}
$$

By comparing with (3.45), we see that this algebra is isomorphic to the matrix algebra generated by $\mathbf{z}$ and $\mathbf{z}^{\dagger}$ in the $2 \times 2$ representation. $z_{F}$ and $\bar{z}_{F}$ can be written as

$$
z_{F}=\frac{\lambda_{\frac{1}{2},-\frac{1}{2}}}{8} \mathcal{Z}_{01}, \quad \bar{z}_{F}=\frac{\lambda_{\frac{1}{2},-\frac{1}{2}}}{8} \mathcal{Z}_{10}
$$

and for their star products we get

$$
z_{F} \star \bar{z}_{F}=\frac{\lambda_{\frac{1}{2},-\frac{1}{2}}^{2}}{8} \mathcal{Z}_{00}, \quad \bar{z}_{F} \star z_{F}=\frac{\lambda_{\frac{1}{2},-\frac{1}{2}}}{8} \mathcal{Z}_{11},
$$

along with $z_{F} \star z_{F}=\bar{z}_{F} \star \bar{z}_{F}=0$. (This is in contrast with the ordinary product which gives $\left.\left|z_{F}\right|^{2}=\left(\lambda_{\frac{1}{2},-\frac{1}{2}} / 8\right)^{2} \mathcal{Z}_{00} \mathcal{Z}_{11} \cdot\right)$

Finally, we return to the stereographic projection. For any $j$ we can write it (or more precisely, the inverse stereographic projection) in terms of $N_{j}(x)$. Here we invert (3.41) to 
solve for the three dependent coordinates $\left(x_{i}\right)_{F}=\left\langle\zeta\left|\mathbf{x}_{i}\right| \zeta>\right.$ of the fuzzy sphere. After using $\mathbf{x}_{3}=\alpha(\mathbf{n}-j)$,

$$
\begin{aligned}
\left(x_{3}\right)_{F} & =\alpha \sum_{n=0}^{2 j} \frac{n-j}{n !\left(\left[f_{j}(n)\right] !\right)^{2}} \mathcal{Z}_{n n} \\
& =\alpha\left[\zeta \frac{\partial}{\partial \zeta} \ln N_{j}\left(|\zeta|^{2}\right)-j\right]
\end{aligned}
$$

while

$$
\begin{aligned}
&\left(x_{1}\right)_{F}-i\left(x_{2}\right)_{F}=z_{F} \star\left(1-\left(x_{3}\right)_{F}\right) \\
&=\sum_{n=0}^{2 j-1} \frac{1-\alpha(n+1-j)}{n !\left(\left[f_{j}(n)\right] !\right)^{2}} \mathcal{Z}_{n n+1} \\
&=(1+\alpha(j-1)) \zeta-\alpha|\zeta|^{2} \frac{\partial}{\partial \bar{\zeta}} \ln N_{j}\left(|\zeta|^{2}\right)+\frac{\alpha(j+1)-1}{(2 j) !\left(\left[f_{j}(2 j)\right] !\right)^{2}} \mathcal{Z}_{2 j} 2 j+1 \\
&=\sum_{n=0}^{2 j-1} \frac{1-\alpha(n+1-j)}{n !\left(\left[f_{j}(n)\right] !\right)^{2}} \mathcal{Z}_{n+1 n} \\
&\left(x_{1}\right)_{F}+i\left(x_{2}\right)_{F}=\left(1-\left(x_{3}\right)_{F}\right) \star \bar{z}_{F} \\
&=(1+\alpha(j-1)) \bar{\zeta}-\alpha|\zeta|^{2} \frac{\partial}{\partial \zeta} \ln N_{j}\left(|\zeta|^{2}\right)+\frac{\alpha(j+1)-1}{(2 j) !\left(\left[f_{j}(2 j)\right] !\right)^{2}} \mathcal{Z}_{2 j+12 j}
\end{aligned}
$$

It identically follows that

$$
\left(x_{1}\right)_{F} \star\left(x_{1}\right)_{F}+\left(x_{2}\right)_{F} \star\left(x_{2}\right)_{F}+\left(x_{3}\right)_{F} \star\left(x_{3}\right)_{F}=1 .
$$

The right hand sides of (3.65 3.67) can in principle be reexpressed in terms of the stereographic coordinates $z_{F}$ and $\bar{z}_{F}$. For instance, for the case of $j=\frac{1}{2}$ we have the result simple results:

$$
\begin{gathered}
\left(x_{3}\right)_{F}=\frac{\bar{z}_{F} \star z_{F}-z_{F} \star \bar{z}_{F}}{2 \sqrt{3}(2+\sqrt{3})} \\
\left(x_{1}\right)_{F}-i\left(x_{2}\right)_{F}=\left(1-\frac{1}{\sqrt{3}}\right) z_{F}, \quad\left(x_{1}\right)_{F}+i\left(x_{2}\right)_{F}=\left(1-\frac{1}{\sqrt{3}}\right) \bar{z}_{F}
\end{gathered}
$$

We can check that the standard inverse stereographic projection is recovered in the commutative limit. Substituting (3.56) into (3.65 3.67) gives

$$
\begin{gathered}
\left(x_{3}\right)_{F} \rightarrow \frac{|\zeta|^{2}-1}{|\zeta|^{2}+1} \\
\left(x_{1}\right)_{F}-i\left(x_{2}\right)_{F} \rightarrow \frac{2 \zeta}{|\zeta|^{2}+1}, \quad\left(x_{1}\right)_{F}+i\left(x_{2}\right)_{F} \rightarrow \frac{2 \bar{\zeta}}{|\zeta|^{2}+1} .
\end{gathered}
$$


Since $(\zeta, \bar{\zeta})$ and $\left(z_{F}, \bar{z}_{F}\right)$ coincide in the limit they both reduce to the usual stereographic coordinates $(z, \bar{z})$ of the commutative sphere in this limit. (A different deformation of the standard stereographic projection can be found in [28].)

Acknowledgement We are gratefull for valuable discussions with A. P. Balachandran, G. Karatheodoris, D. O'Connor and C. Zachos (who also informed us of numerous references). This work was supported by the joint NSF-CONACyT grant E120.0462/2000. G. A. was supported in part by the U.S. Department of Energy under contract number DE-FG02-85ER40231,

and A. P. and A. S. were supported in part by the U.S. Department of Energy under contract number DE-FG05-84ER40141.

\section{Appendix A}

Here we obtain the expression (3.53) for $N_{j}(x)$ for the fuzzy sphere, and give its asymptotic expansion (3.56).

From (3.50),

$$
\left(\left[f_{j}(n)\right] !\right)^{2}=\frac{(2 j+1) ! \Gamma(\sqrt{j(j+1)}+j-n)^{2}}{(2 j-n) ! \Gamma(\sqrt{j(j+1)}+j+1)^{2}} .
$$

Substituting into (3.52) gives

$$
N_{j}(x)=\frac{\Gamma(\gamma+2 j+1)^{2}}{\Gamma(2 j+2)} \sum_{n=0}^{2 j} \frac{\Gamma(2 j-n+1)}{\Gamma(\sqrt{j(j+1)}+j-n)^{2}} \frac{x^{n}}{n !},
$$

where $\gamma=\sqrt{j(j+1)}-j$. Now replace the summation index by $m=2 j-n$ to get

$$
N_{j}(x)=\frac{\Gamma(\gamma+2 j+1)^{2}}{\Gamma(2 j+2)} x^{2 j} \sum_{m=0}^{2 j} \frac{\Gamma(m+1)^{2}}{\Gamma(2 j+1-m) \Gamma(\gamma+m)^{2}} \frac{x^{-m}}{m !} .
$$

From the identity

$$
\frac{\Gamma(a+1)}{\Gamma(a-m)}=-(-1)^{m} \frac{\Gamma(m+1-a)}{\Gamma(-a)}
$$

it follows that

$$
N_{j}(x)=-\frac{\Gamma(\gamma+2 j+1)^{2}}{\Gamma(-2 j-1) \Gamma(2 j+2)^{2}} x^{2 j} \sum_{m=0}^{2 j} \frac{\Gamma(m-2 j) \Gamma(m+1)^{2}}{\Gamma(\gamma+m)^{2}} \frac{(-x)^{-m}}{m !} .
$$

This is just (3.53), once we use the definition

$$
{ }_{3} F_{2}\left(a_{1}, a_{2}, a_{3} ; b_{1}, b_{2} ; x\right)=\frac{\Gamma\left(b_{1}\right) \Gamma\left(b_{2}\right)}{\Gamma\left(a_{1}\right) \Gamma\left(a_{2}\right) \Gamma\left(a_{3}\right)} \sum_{n=0}^{\infty} \frac{\Gamma\left(a_{1}+n\right) \Gamma\left(a_{2}+n\right) \Gamma\left(a_{3}+n\right)}{\Gamma\left(b_{1}+n\right) \Gamma\left(b_{2}+n\right)} \frac{x^{n}}{n !} .
$$

The infinite series expression is valid provided no $a_{i}$ are negative integers, which excludes our case. On the other hand, the series terminates when $a_{i}$ is a negative integer, say $-2 j$ (our case), 
at $n=2 j$, and the hypergeometric function (A.5) is then referred to as an extended Laguerre polynomial. To find the asymptotic representation for large $j$ one can use the Darboux analysis [27. For the extended Laguerre polynomials the result up to $1 / j^{2}$ corrections is

$$
\begin{array}{r}
{ }_{3} F_{2}\left(a_{1}, a_{2},-2 j ; b_{1}, b_{2} ;-x\right) \sim \frac{\Gamma\left(b_{1}\right) \Gamma\left(b_{2}\right)}{\Gamma\left(a_{1}\right) \Gamma\left(a_{2}\right)}(1+x)^{2 j}\left(\frac{2 j x}{1+x}\right)^{\beta} \times \\
\exp \left\{-\frac{u}{2 j x}+\frac{v}{2 j}+O\left(j^{-2}\right)\right\}+O\left(j^{-2}\right),
\end{array}
$$

where $0<x \ll 2 j$ and

$$
\begin{aligned}
& \beta=a_{1}+a_{2}-b_{1}-b_{2} \\
& u=a_{1} a_{2}-b_{1} b_{2}+\beta\left(1-a_{1}-a_{2}\right) \\
& v=-a_{1} a_{2}+b_{1} b_{2}+\frac{1}{2} \beta\left(a_{1}+a_{2}+b_{1}+b_{2}-1\right) .
\end{aligned}
$$

Using $\beta=2(1-\gamma), u=-(1-\gamma)^{2}$ and $v=\gamma(1-\gamma)$, we get (3.56).

\section{REFERENCES}

[1] H. Groenewold, Physica (Amsterdam) 12, 405 (1946).

[2] J. Moyal, Proc. Camb. Phil. Soc. 45, 99 (1949).

[3] C. Zachos, J. Math. Phys. 41, 5129 (2000); hep-th/0008010, to be published in the proceedings of NATO Advanced Research Workshop on Integrable Hierarchies and Modern Physical Theories (NATO ARW - UIC 2000), Chicago, Illinois, 22-26 Jul 2000.

[4] H. Grosse and P. Presnajder, Lett.Math.Phys. 28, 239 (1993); P. Presnajder, hep$\operatorname{th} / 9912050$.

[5] J. Madore, Class. and Quant. Geom. 9, 69 (1992); An Introduction to Noncommutative Differential Geometry and its Applications, Cambridge University Press, Cambridge, 1995.

[6] U. Carow-Watamura, S. Watamura, Commun. Math. Phys. 183, 365 (1997); Int. J. Mod. Phys. A13, 3235 (1998); Commun. Math. Phys. 212, 395 (2000).

[7] S. Baez, A.P. Balachandran, B. Idri, S. Vaidya, Commun.Math.Phys. 208, 787 (2000).

[8] A.P. Balachandran, T.R. Govindarajan, B. Ydri, hep-th/9911087; hep-th/0006216.

[9] A.P. Balachandran, S. Vaidya, hep-th/9910129.

[10] A.P. Balachandran, X. Martin, D.O'Connor, hep-th/0007030.

[11] A. Alekseev, A. Recknagel, V. Schomerus, JHEP 0005:010,2000; P.-M. Ho, hepth/0010165. 
[12] A. Perelomov, Generalized Coherent States and Their Applications (Springer-Verlag, Berlin, 1986); J.R. Klauder and B.-S. Skagerstam Coherent States (World Scientific, Singapore, 1985).

[13] M. Kontsevich, q-alg/9709040.

[14] F.A. Berezin, Commun.Math.Phys. 40153 (1975).

[15] V.I. Man'ko, G. Marmo, E.C.G. Sudarshan, F. Zaccaria, Physica Scripta 55, 528 (1997).

[16] F. Bayen, in Group Theoretical Methods in Physics, ed. E. Beiglböck, et. al. [Lect. Notes Phys. 94, 260 (1979)]; A. Voros, Phys. Rev. A 40, 6814 (1989).

[17] N. Read, Phys.Rev. B58, 16262 (1998).

[18] V. Dodonov and V. Man'ko, Physica 137A, 306 (1986).

[19] T. Curtright, T. Uematsu and C. Zachos, to appear.

[20] P. Podles, Lett. Math. Phys. 14, 193 (1987).

[21] T. Holstein and H. Primakoff, Phys.Rev. 58, 1098 (1940).

[22] R. Gray and C.A. Nelson, J. Phys A23, (1990) L945; C.A. Nelson, Contributed to 6th Symposium on Symmetries in Science: From Rotation Group to Quantum Algebras, Bregenz, Austria, 2-7 Aug 1992. In *Bregenz 1992, Proceedings, Symmetries in science $6 *$ 523-535; C.A. Nelson and M. Fields, Phys Rev A51, (1995) 2410.

[23] M. Arik and D.D. Coon, J. Math. Phys. 17, (1976) 524; E. Celeghini, M. Rasetti and G. Vitiello, Phys. Rev. Lett. 66, (1991) 2056; J. Katriel and A. Solomon, J. Phys. A24, (1991) 2093; Jurco, Lett. Math. Phys. 21 (1991) 51; V. Buzek, J. Mod. Optics 38, (1991) 801; L.-M. Kuang and F.-B. Wang, Phys. Lett. A173, (1993) 221; L.-M. Kuang and F.-B. Wang, J. Phys. A26, (1993) 293; Y. Yang and Z. Yu, Mod. Phys. Lett. A9, (1994) 3367.

[24] A. Klimyk and K. Schmüdgen, Quantum Groups and Their Representations (SpringerVerlag, Berlin, 1997).

[25] L.C. Biedenharn, J. Phys. A22, L873 (1989).

[26] A.J. MacFarlane, J. Phys. A22, 4581 (1989).

[27] Y.L. Luke, The Special Functions and Their Approximations, vol. 1 (Academic Press, New York and London, 1969).

[28] T. Hakioglu and M. Arik, Phys. Rev. A54, 52 (1996). 